\title{
A 200 años de la Constitución de 1819. Breve comentario de su historia
}

200 years after the Constitution of 1819. Brief comment on its bistory

200 anos após a Constituição de 1819. Breve comentário sobre sua história 200 années après la Constitution de 1819. Un bref commentaire de son bistoire

Diego Gabriel Presa ${ }^{1}$ | Universidad Nacional de La Plata

Revista Derechos en Acción ISSN 2525-1678/ e-ISSN 2525-1686

Año 4/No 11 Otoño 2019 (21 marzo a 21 junio), 357-372

DOl: https://doi.org/10.24215/25251678e280

ORCID: https://orcid.org/0000-0003-3315-9301

Recibido: 04/05/2019

Aprobado: 27/05/2019

Resumen: El trabajo aborda históricamente la Constitución de 1819, dando cuenta del contexto en el que fue sancionada. Se explica que nació muerta por distintos motivos. Uno de ellos es que el gobierno encabezado por el Directorio estaba totalmente desacreditado y sin consenso hacia el interior de la Republica o las restantes 13 provincias, (14 si tenemos presente que la Banda Oriental se encuentra ocupada por los portugueses), este descrédito hizo de la Constitución, un proyecto de laboratorio que no tenía ningún consenso de fuerzas políticas y no reflejaba la realidad de las Provincias Unidas y sus intereses. Este proceso constitucional dejo una enseñanza, siempre que se quiera redactar una constitución que va a regir en la vida de los ciudadanos debe hacerse en el mayor consenso posible, de lo contrario estará destinado al fracaso.

Palabras claves: Constitución. Historia.

1 Abogado, adjunto cátedra III de Historia Constitucional, Facultad de Ciencias Jurídicas y Sociales, UNLP. 
Abstract: This work addresses historically the Constitution of 1819, recounting the context in which it was sanctioned. It is explained that she was born dead for different reasons. One of them is that the government headed by the Directory was totally discredited and without consensus towards the interior of the Republic or the remaining 13 provinces (14 if we take into account that the Banda Oriental is occupied by the Portuguese). This discredits turned the Constitution into laboratory project that had no consensus of the political forces and did not reflect the reality of the United Provinces and their interests. This constitutional process has left us a teaching: whenever a constitution that will rule the life of citizens needs to be written, it must be made in the greatest possible consensus, otherwise it will be destined to failure.

Keywords: Constitution. History

Resumo: 0 trabalho aborda historicamente a Constituição de 1819, dando conta do contexto em que foi sancionada. Explica-se que ela nasceu morta por diferentes razões. Uma delas é que o governo encabeçado pelo Diretório estava totalmente desacreditado e sem consenso no interior da República ou das 13 províncias restantes, (14 se tivermos presente que a Banda Oriental encontra-se ocupada pelos portugueses), esse descrédito fez da Constituição, um projeto de laboratório que não tinha consenso de forças políticas e não refletia a realidade das Províncias Unidas e seus interesses. Este processo constitucional deixou um ensinamento, sempre que quiser escrever uma constituição que irá governar na vida dos cidadãos deve ser feita no maior consenso possível, caso contrário será destinado ao fracasso.

Palavras-chave: Constituição. História

Résumé: Ce travail aborde historiquement la Constitution de 1819 en rendant compte du contexte dans lequel il a été sanctionné. On explique qu'elle est née morte pour différentes raisons, l'une desquels est que le gouvernement dirigé par le directoire était totalement discrédité et sans consensus vers l'intérieur de la République ou les 13 provinces restantes (14 si l'on se souvient que la bande orientale est occupée par les Portugais). Ce discrédit a fait de la Constitution un projet de laboratoire sans consensus des forces politiques et qui ne reflétait pas la réalité et les intérêts des Provinces-Unies. Cette procédure constitutionnelle a laissé un enseignement, chaque fois qu'on veut élaborer une constitution 
qui va régir la vie des citoyens, on a besoin de le plus grand consensus possible, autrement elle sera vouée à l'échec.

Mot-clés: Constitution. Histoire

\section{Introducción}

El 22 de abril de 1819 el Congreso de Tucumán, sesionaba en Buenos Aires y sanciona el primer texto constitucional orgánico para las Provincias Unidas del Rio de La Plata el Estado; texto que no van a aceptar las provincias litorales de la Banda Oriental del Uruguay -no solo por no coincidir con el mismo, sino por estar ocupada por los portugueses desde 1816 a instancias de Buenos Aires- y las provincias de Corrientes, Entre Ríos y Santa Fe.

No obstante este texto constitucional, que es el primero en su estilo, llevara al camino a la Constitución de 1853.

Hoy doscientos años después podemos recorrer su articulado y ver dicho texto en profundidad, donde muchas de las atribuciones de los poderes del estado, se fueron manteniendo en los textos constitucionales que le sucedieron, hasta terminar en artículos vigentes en la Constitución Nacional de 1853 y sus reformas.

Nos sorprende que no tenga la organización de la que dispondrán los constituyentes de 1853. Si contiene una estructura de gobierno representativo, republicano, adhiriendo tácitamente al sistema unitario, pero sin mencionarlo; sólo se desprenderá de su articulado y formato, que es unitaria.

Además de su texto se desprende, una actitud altamente conservadora, con tendencias aristocráticas en la composición de la Honorable Cámara de Senadores. Esta situación tan particular se da por las negociaciones autorizadas por el mismo Congreso al Director Supremo, de buscar un príncipe o princesa europea que asumiera como Jefe de Estado de las Provincias Unidas del Rio de La Plata. 
Recordemos que existieron cuatro proyectos que pueden identificarse:

1) La princesa Carlota Joaquina hija de Carlos IV de España, hermana del rey Fernando VII y esposa del rey de Portugal, que se encontraba en Río de Janeiro, Brasil.

2) Francisco de Paula hijo de Carlos IV y hermano de Fernando VII.

3) Luis Felipe de Orleans, Duque de Orleans, quien con el tiempo sería coronado como rey de Francia

4) Carlos Luis de Borbón, duque de Luca, sobrino de Fernando VII.

5) el proyecto incaico que detentó tres candidatos:

a) Dionisio Inca Yupanqui, educado en el Seminario de Nobles, coronel del regimiento de Dragones y diputado a las Cortes representando al Perú.

b) Juan Andrés Ximénez de León Manco Capac, descendiente legítimo de los emperadores del Perú, sacerdote y primer capellán del ejército argentino.

c) Juan Bautista Túpac Amarú, quinto nieto del último emperador del Perú, quien en 1816 hacía treinta y cinco años que se encontraba prisionero de los españoles.

La constitución de 1819 estaba destinada a albergar alguno de estos proyectos monárquicos, que se vieron fracasados por distintas circunstancias históricas.

La definición de Constitución, se entiende, "como el conjunto de normas, que reconoce derechos y garantías para sus ciudadanos y regula el funcionamiento de los poderes del Estado".

El texto de 1819 no empieza por las Declaraciones, Derechos y Garantías, pero con el fin de mantener el formato de nuestro actual texto constitucional empezaré por ellas. 


\section{II. "Declaraciones Derechos y Garantías"}

A diferencias de la Constitución de 1853, la Constitución de 1819, en su sección Quinta, estableció la declaración de derechos dividido en dos capítulos: el primero sobre los "Derechos de la Nación" y el segundo sobre "Derechos Particulares".

Podemos distinguir los siguientes:

Los derechos de la Nación regulados entre los artículos 104 a 108, del Capítulo I:

Así, el artículo 104 le da el derecho de reformar la constitución cuando lo "exija el interés común"

El artículo 105 establece la soberanía -como poder- en los tres poderes del estado, fijando claramente la división de poderes.

El artículo 106 y 107 establece las responsabilidades de los funcionarios ante el Estado y que ninguna autoridad está por encima de la ley.

El Artículo 108 habla de la delegación de esa soberanía constitucional, le permite a la Nación la facultad de nombrar a sus Representantes, y la de "ejercer libremente el poder censorio por medio de la prensa."

En el Capítulo II, se establecen los Derechos Particulares, regulados entre los artículos $\mathbf{1 0 9}$ a $\mathbf{1 2 9}$.

El Artículo 109 fija que, los miembros del Estado deben ser protegidos en el goce de los derechos de su vida, reputación, libertad, seguridad y propiedad.

El Artículo 110, establece la igualdad ante la ley, diciendo textualmente "...favorecer igualmente al poderoso que al miserable para la conservación de sus derechos..."

La libertad de prensa se regula en el artículo 111 diciendo que "...es un derecho tan apreciable al hombre como esencial para la conservación de la libertad civil en un Estado...", una ley del Congreso debía regular la misma.

El derecho a la intimidad se establece en el artículo 112, “...Las acciones privadas de los hombres que de ningún modo 
ofenden el orden público, ni perjudican a un tercero, están solo reservadas a Dios, y exentas de la autoridad de los Magistrados...", actualmente este mismo texto está consagrado en el artículo 19 de la Constitución Nacional. El mismo, se ve reflejado en el artículo 113 “...Ningún habitante del Estado, será obligado a hacer lo que no manda la ley, ni privado de lo que ella no prohíbe...".

El juez natural se regula en el Artículo 114, así como el juicio por jurados, en cuanto lo permitan las circunstancias. El artículo 115 protege a los ciudadanos de las requisiciones arbitrarias dejando a la regulación de la ley dichos procedimientos.

Los artículos 116 y 118 establecen el principio de inocencia, el debido proceso legal, siendo que ningún habitante del Estado puede ser penado, ni confinado, sin que preceda forma de proceso y sentencia legal.

Estos últimos artículos conjuntamente con el Artículo 117, hoy se encuentran regulados en el artículo 18 de la Constitución Nacional, se consagra que las cárceles solo deben servir para la seguridad y no para castigo de los reos.

La protección del domicilio se fijó en el artículo 119 y solo podrá allanarse en caso de resistencia a la autoridad legítima. El artículo 120 entiende que la autoridad legítima es el juez. A mayor protección el artículo $\mathbf{1 2 1}$ determina que las anteriores disposiciones relativas a la seguridad individual no podrán suspenderse.

El Artículo 123 vuelve hablar de la propiedad como un derecho sagrado e inviolable, y que los miembros del Estado no pueden ser privados de ella, ni gravados en sus facultades sin el consentimiento del Cuerpo Legislativo, o por un juicio conforme a las leyes. Regulando la expropiación en el artículo 124.

Por los tiempos de guerra tanto de la Independencia como la Guerra Civil el Artículo 125 hablaba de los auxilios que no estaban obligados a prestar los ciudadanos a los ejércitos, sino por orden del magistrado civil, siendo indemnizados por los daños.

El derecho a peticionar se fija en el artículo 126. 
Este artículo 127, es un antirreflejo del actual 16 de la Constitución Nacional y se prohibían nuevos títulos de nobleza hereditaria, entendiéndose la existencia de los títulos de nobleza que la propia Asamblea del año XIII había abolido.

Los pueblos originarios son contemplados en el artículo 128 fijando una igualdad que se vuelve distintiva, porque los iguala a los ciudadanos de las Provincias Unidas, siendo que ya eran integrantes de estas Provincias Unidas. Vuelve a abolir o reforzar cualquier tipo de tributo o tasa o servicio personal, además le da facultados al Congreso para promover políticas legales que mejoren las condiciones de vida.

El artículo 129, va más allá de lo fijados por la Asamblea del año XIII, aboliendo el tráfico de esclavos y prohibida para siempre su introducción en el territorio del Estado, no quedando claro si la esclavitud en sí, dejaba de existir en el territorio de las Provincias Unidas, recordemos que la Constitución de 1853 abole la misma y declara libres a los pocos que aún existen.

\section{Los Poderes del Estado}

La constitución en este sentido es clásica y divide los poderes en Ejecutivo, Legislativo y Judicial

La Sección Segunda, en su artículo 3, dice que las facultades legislativas eran ejercidas por un Congreso Nacional, compuesto de dos Cámaras, una de Representantes y otra de Senadores.

En el Capítulo I, y a partir del artículo 4 al 9 se encuentra la Cámara de Representantes o Diputados, elegidos una cada 25 mil o fracción de 16.500 .

Entre los requisitos el artículo $5^{\circ}$, siete años de ciudadano antes de su nombramiento, 26 años de edad cumplidos, un fondo de cuatro mil pesos o arte, profesión u oficio útil.

Durarían cuatro años, pero se renovarán por mitad al fin de cada bienio. Sorteando la mitad de la Cámara a renovar en la primera renovación (art 6). 
La Cámara de Representantes (Art. $7^{\circ}$ ) tiene exclusivamente la iniciativa en materia de contribuciones, tasas e impuestos. Además del derecho exclusivo (art $8^{\circ}$ ) de acusar de oficio, o a instancia de cualquier ciudadano, a los miembros de los tres grandes poderes, a los Ministros de Estado, Enviados a las Cortes Extranjeras, Arzobispos u Obispos, Generales de los ejércitos, Gobernadores y Jueces de las Provincias, y demás empleados de no inferior rango a los nombrados, por los delitos de traición, concusión, malversación de los fondos públicos, infracción de Constitución, u otros que según las leyes merezcan pena de muerte o infamia, es lo que se denomina juicio político, donde la Cámara de Representante o diputados hace de fiscal.

En cuanto a los impuestos, tasas y contribuciones, son exclusivas de la Cámara por ser los representantes del pueblo de la Nación.

En cuanto a las retribuciones -sueldos- de los representantes los fija la propia cámara según el artículo $9^{\circ}$.

El capítulo II, habla del Senado, entre los artículos 10 a 20, aquí la situación es más compleja por la composición del mismo.

Según el artículo 10 lo conformaran un senador por Provincia, tres senadores militares, cuya graduación no baje de Coronel Mayor; un Obispo, y tres Eclesiásticos; un Senador por cada Universidad; y el Director de Estado, concluído el tiempo de su Gobierno.

Entre los requisitos para ser Senador, la edad de treinta años cumplidos, nueve de ciudadano antes de su elección, un fondo de ocho mil pesos, una renta equivalente, o una profesión que lo ponga en estado de ser ventajoso a la sociedad (art. 11). Durán en el cargo doce años, renovándose por terceras partes cada cuarto. La suerte decidirá quiénes deban salir en el primer y segundo cuatrienio (art. 12).

El ex Director Supremo o saliente integra el Senado hasta que sea reemplazado por el que le sucediese en el mando (art. 13). 
La elección de los Senadores de las Provincias, se hará en cada Municipalidad nombrará un capitular y un propietario, que tengan un fondo de diez mil pesos al menos, para electores.

Reunidos éstos en un punto en el centro de la Provincia, que designará el Poder Ejecutivo, elegirán tres sujetos de la clase civil, de los que uno al menos sea de fuera de la Provincia. Esta terna se pasará al Senado (la primera vez al Congreso) con testimonio íntegro del acta de elección.

El Senado, recibidas todas las ternas y publicadas por la prensa, hará el escrutinio; y los que tuvieren el mayor número de sufragios, computados por las Provincias, serán Senadores. Si no resultase pluralidad, la primera vez el Congreso, y en los sucesivo el Senado, hará la elección entre los propuestos.

Así lo establecía el artículo 14 la elección, la cual es de formato indirecta al no participar el pueblo en su elección.

El articulo 15 habla de los Senadores militares que eran nombrados por el Director de Estado.

Los Obispos; en el artículo 16, regula la elección siendo Senador por primera vez el Obispo de la Diócesis donde reside el Cuerpo Legislativo. En lo sucesivo se elegirá el Obispo Senador por los Obispos del territorio, remitiendo sus votos al Senado. Publicados por la prensa, se hará el escrutinio, y el que reuniese el mayor número, será Senador: no resultando pluralidad, decidirá la elección el Senado.

En cuanto a los eclesiásticos en el artículo 17, fijaba que los Cabildos eclesiásticos, reunidos por el prelado Diocesano, Curas Rectores del Sagrario de la Iglesia Catedral, y Redactores de los Colegios (cuando éstos sean eclesiásticos) elegirán tres individuos del mismo estado, de los cuales, uno al menos sea de otra Diócesis. Remitidas y publicadas las ternas con sus actas, los tres que reúnan mayor número de sufragios computados por las iglesias, serán Senadores; en caso de igualdad el Congreso o Senado decidirá la elección.

Al igual que en la actual Constitución Nacional el articulo 18 le da al Senado la función de juez en el juicio político. 
Necesitando según el Artículo 19 la concurrencia de dos terceras partes de sufragios para dictar sentencia, no establece si son de senadores presentes o totales del cuerpo.

El artículo 20 deja al destituído a disposición de la ley común.

En cuanto a las atribuciones de ambas Cámaras el Capítulo III, las regula del artículo 21 a 30, entre las que encontramos en el artículo 21 los meses de sus sesiones en los meses de Marzo, Abril y Mayo, Septiembre, Octubre y Noviembre.

Cada Cámara es Juez de los títulos y elección de sus miembros (Art. 22), por el artículo 23 Nombra sus autoridades así como su reglamento interno. En cuanto a su funcionamiento es por quórum, no pudiendo hacerlo sin ello (art 24), sus sesiones serán registradas en un diario (Art. 25), tienen fueros durante su asistencia a la Legislatura, y mientras van y vuelven de ella: excepto el caso de ser sorprendidos in fraganti en la ejecución de algún crimen que merezca pena de muerte, infamia u otra aflictiva (art 26); e iguales fueros por sus opiniones (Art. 27); Cuando se forme querella -denuncia- por escrito contra cualquier Senador o Representante, por delitos, examinado el mérito del sumario en juicio público, podrá cada Sala con dos tercios de votos separar al acusado de su seno, y ponerlo a disposición de la Justicia, para su enjuiciamiento (art 28); la incompatibilidad de un cargo legislativo y del Ejecutivo sin la autorización de la Cámara respectiva (Art. 29); la posibilidad de interpelar a los Ministros del Poder Ejecutivo para recibir los informes que estime convenientes (art 30).

\section{En el Capítulo IV, se encuentran las atribuciones del congreso}

a) $\mathrm{Al}$ Congreso corresponde privativamente formar las leyes que deben regir en el territorio de la Unión (art 31); b) Art. 32 Decretar la guerra y la paz. Art. 33 Establecer derechos; y contribuciones proporcionalmente iguales en todo el territorio., fijando un plazo de 2 años de vigencia de ellas; c) Art. 34 Fijar 
a propuesta del Poder Ejecutivo la fuerza de línea de mar y tierra; d) Art. 35 Mandar construir y equipar una marina nacional. e) Art. 36 Recibir empréstitos sobre los fondos del Estado; f) Art. 37 Reglar la forma de todos los juicios, y establecer Tribunales inferiores a la Alta Corte de Justicia. g) Art. 38 Crear y suprimir empleos de toda clase. h) Art. 39 Reglar el comercio interior y exterior. i) Art. 40 Demarcar el territorio del Estado, y fijar los límites de la Provincias. j) Art. 41 Habilitar puertos nuevos y elevar las poblaciones al rango de villas, ciudades o Provincias. k) Art. 42 Formar planes uniformes de educación pública, y proveer de medios para el sostén de los establecimientos de esta clase. 1) Art. 43 Recibir anualmente del Poder Ejecutivo la cuenta general de las rentas públicas, examinarlas y juzgarlas; 11) Art. 44 Asegurar a los autores o inventores de establecimientos útiles, privilegios exclusivos por tiempo determinado; m) art. 45 Reglar la moneda, los pesos y medidas.

Las atribuciones no difieren de las que hoy tiene el actual Congreso, pero teniendo presente que hoy en el artículo 75 de la CN., esas atribuciones poseen la materia delegada de los Estados provinciales en su inciso 12 en cuanto a los códigos de fondo.

En cuanto a la formación y sanción de las leyes el Capítulo V, lo regula entre los artículos 46 a 55, siendo la regla general que pueden tener inicio en cualquiera de las dos Cámaras, exceptuando las del artículo $7^{\circ}$ de la Constitución.

Curiosamente cuando entramos a temas específicos el art. 48 , establece que cada proyecto de ley se leerá en tres sesiones distintas, mediando entre cada una de ellas tres días al menos: sin esto no se pasará a deliberar, siendo esta una regla de funcionamiento interno que debería estar en el reglamento de las cámaras y no en el texto constitucional.

Para su aprobación el artículo 49 establece que la mitad más uno de sufragios en cada una de la Cámaras constitucionalmente reunidas. Aprobado el proyecto, se pasará a la otra para 
su discusión (Art 50). Si el proyecto es desaprobado no podrá tener tratamiento dentro de ese año legislativo, quedando postergado para el año legislativo entrante (Art. 51); Los proyectos de ley aprobados, pasarán al Director del Estado (art 52). Si él -Director- los subscribe, o en el término de quince días no los devuelve objetados, tendrán fuerza de ley. (Art 53). La objeción es un veto, que es descripto en el artículo 54 devolviendo el proyecto a donde tuvo su origen. Si ambas Cámaras, con dos tercios de sufragios en cada una de ellas, insistieran en el proyecto vetado se sancionaba.

El Poder Ejecutivo, está en la sección tercera, Capítulo I, con el título de "Naturaleza y calidades de este Poder"; El Poder Ejecutivo de la Nación es ejercido por un Director (art 56); para ser Director requería seis años de residencia y treinta y cinco de edad (Art. 57). Tampoco podrá ser elegido el que se halle empleado por el Senado o en la Cámara de Representantes (Art. 58), se le toma juramento de manos del Presidente del Senado, en presencia de las dos Cámaras reunidas, es decir en Asamblea legislativa (art 59); y duraba en el cargo cinco años (art 60); En caso de enfermedad, acusación o muerte del Director del Estado, administrará provisoriamente el Poder Ejecutivo el Presidente del Senado, quedando entretanto suspenso de las funciones de Senador (art 61).

En cuanto a la forma de elección del Director del Estado, fue regulado en el Capítulo II, era elegido por las dos Cámaras reunidas (art 62); presidida la elección por el Presidente del Senado, siendo Vicepresidente el Presidente de la Cámara de Representantes (art 63), los votos se entregarán escritos y firmados por los vocales, y se publicarán con sus nombres (art 64); la mayoría será de mitad más uno de cada Cámara, hará la elección (art 65).

En caso de tres votaciones y si ninguno obtuviese la expresada mayoría, se publicarán los tres sujetos que hayan obtenido el mayor número, y por ellos solos se sufragará en las siguientes votaciones (art 66); previendo el art. 67 si reiterada ésta hasta tres veces, ninguno de los tres propuestos reuniese la mayoría 
que exige el artículo 65, se excluirá el que tuviese menor número de votos: caso de igualdad entre los tres o dos de ellos, decidirá la suerte el que haya de ser excluido, quedando solamente dos; definiéndose en el art. $\mathbf{6 8}$ por uno de estos. Si repetida la votación, no resultase la mayoría expresada, se sacará por suerte el Director de entre los dos (art 69).

La elección se realizaría 30 días antes de culminar el mandato (art. 71) y en caso de muerte deberá hacerse la elección dentro de quince días. Se permitía una vez la reelección con un voto de las dos terceras partes de cada Cámara (art 73).

En el Capítulo III, se establecen las atribuciones del Poder Ejecutivo, entre las que encontramos, Jefe Supremo de todas las fuerzas de mar y tierra (art 74); de publicar y hace ejecutar las leyes (art 75); Realizaba la apertura de las sesiones del Cuerpo Legislativo, informando en esta ocasión sobre el estado de Gobierno (art 76); Convoca a sesiones extraordinarias (art 77); puede proponer proyectos a las Cámaras (art 78); Rechaza las invasiones de los enemigos exteriores: previene las conspiraciones y sofoca los tumultos populares (art 80); Nombra por sí solo los Generales de los ejércitos de mar y tierra, los Embajadores, Enviados y Cónsules en las naciones extranjeras, y los recibe (art 81); Nombra y destituye a sus Ministros (art 82); puede celebrar y concluir tratados con las naciones extranjeras: salvo el caso de enajenación o desmembración de alguna parte del territorio, en que deberá exigirse el dos tercios de la Cámara de Representantes (art 83); Expide las cartas de ciudadanía de acuerdo a la ley (art 84); Nombra a todos los empleos (art 85); Nombra a los Arzobispos y Obispos, a propuesta en terna del Senado ejerciendo aquí el derecho de patronato (art 86); Puede indultar de la pena capital a un criminal o conmutarla, (art 89); e igualmente lo puede hacer con sentencias de carácter militar (art. 90); Recibía un sueldo que fijaría el Congreso el cual no puede aumentar o disminuir durante su mandato (art. 91), esto muestra la poca volatilidad económica de la época.

En la sección cuarta, se legislo sobre el Poder Judicial, denominado Capítulo Único, Corte Suprema de Justicia, 
paradójicamente se la llamo Alta Corte de Justicia, compuesta de siete Jueces y dos Fiscales, (art 92); requisito ineludible ser abogado, con ocho años de ejercicio público como tal, y cuarenta años de edad (art 93); Los miembros de la Alta Corte de Justicia, eran nombrados por el Director del Estado, con noticia y consentimiento del Senado (art 94); El presidente del máximo Tribunal, era electo por un periodo de cinco años a simple pluralidad de sufragios, por sus miembros, (art 95).

En su competencia fijada en el art. 97, entendía exclusivamente de todas las causas concernientes embajadores y cónsules de las naciones extranjeras; de aquellas en que sea parte una Provincia, o que se susciten entre Provincia y Provincia, o pueblos de una misma Provincia, sobre limites u otros derechos contenciosos; de las que tengan su origen de contratos entre el Gobierno Supremo y un particular; y últimamente de las de aquellos funcionarios públicos de que hablan los artículos 20 y 28.

Esta era una competencia originaria y donde el máximo tribunal actuaba como una primera y única instancia.

Asimismo la Alta Corte en el art. 98, conocía en último recurso de los casos que descienden de tratados hechos bajo la autoridad del Gobierno; de los crímenes cometidos contra el derecho público de las naciones, y de todos aquellos en que según las leyes haga lugar a los recursos de segunda suplicación, nulidad o injusticia notoria. Siendo aquí su rol el de un tribunal de alzada de última instancia donde sus sentencias son uniformadoras de la jurisprudencia a los tribunales inferiores.

Los juicios eran públicos y las sentencias se formaban por los votos de cada juez (art 99); Producía un informe en un periodo de tiempo, para el Congreso, de todo lo conveniente para las mejoras de la administración de justicia, (art 100); Cada seis meses recibía de las Cámaras de Justicia una razón exacta de las causas y asuntos despachados en ellas, y de las que quedaban pendientes, (art 101); La inamovilidad de los jueces eran mientras durara su buen comportamiento (art 102); 
El Congreso también fijaba sus sueldos los cuales no podrían ser disminuidos mientras permanezcan en el oficio. Hoy cada poder, fija su propio presupuesto en forma anual.

\section{Conclusión}

La Constitución de 1819 nació muerta por distintos motivos. Uno de ellos es que el gobierno encabezado por el Directorio estaba totalmente desacreditado y sin consenso hacia el interior de la Republica o las restantes 13 provincias, (14 si tenemos presente que la Banda Oriental se encuentra ocupada por los portugueses), este descrédito hizo de la Constitución, un proyecto de laboratorio que no tenía ningún consenso de fuerzas políticas y no reflejaba la realidad de las Provincias Unidas y sus intereses.

El segundo motivo, que debemos tener presente, es que la misma Constitución resulta ser un híbrido en lo que respecta a la forma de gobierno, al no definir claramente que era un estado unitario, pero surge de su texto en forma implícita, no fue casual esta forma de redacción, sino que estaba orientada a las negociaciones de carácter monárquico que se llevaban adelante en el continente Europeo, en resumen la no adhesión a un Gobierno Republicano y de carácter Federal, llevo al fracaso inmediato del texto constitucional.

Esta Constitución fue fuente de la de 1826 y por supuesto de la 1853, pero como la de 1826, dejo una enseñanza, siempre que se quiera redactar un texto constitucional que va a regir en la vida de los ciudadanos debe hacerse en el mayor consenso posible, de lo contrario estará destinado al fracaso.

\section{Referencias bibliográficas}

Badeni, Gregorio, Tratado de Derecho Constitucional, t. I, $1^{\text {a }}$ ed., Bs. As., La Ley, 2004, pp. 108/112.

Dalla Vía, Alberto R., "Las reformas constitucionales", en Susana Albanese; Alberto R. Dalla Vía, Roberto Gargarella et al, 
Derecho Constitucional, $1^{\text {a }}$ ed., Bs. As., Editorial Universidad, 2004, pp. 527/542.

González, Joaquín V., Manual de la Constitución Argentina 1853/1860, $2^{a}$ ed., Bs. As., Estrada, 1983, pp. 57/60.

Presa Diego Gabriel, abogado, adjunto cátedra III de Historia Constitucional, Facultad de Ciencias Jurídicas y Sociales, UNLP. www.saij.gob.ar/docs-f/ediciones/libros/Constituciones_argentinas.pdf Basterra, Marcela Constitución de 1819, Un paso adelante en el proceso de consolidación del Estado Constitucional argentino 